\title{
ON TRANSITIVE AND FULLY TRANSITIVE PRIMARY GROUPS ${ }^{1}$
}

\author{
PAUL HILL
}

Let $G$ be a $p$-primary abelian group and $\beta$ an ordinal number such that $G / p^{\beta} G$ is totally projective. It is shown that $G$ is transitive if and only if $p^{\beta} G$ is transitive. The same result is also proved regarding full transitivity. These theorems extend results of Griffith [1].

The primary group $G$ is said to be potentially transitive if $G /\{x\}$ $\cong G /\{y\}$ whenever $x$ and $y$ are elements of $G$ that have the same height sequence. Using an example suggested by Megibben, we prove there exist primary groups that are not potentially transitive.

1 . Let $G$ be a $p$-primary abelian group. Denote by $p G$ the subgroup of $G$ consisting of all the elements of the form $p x$, where $x$ is in $G$. Define $p^{\alpha} G$ inductively by $p^{\alpha+1} G=p\left(p^{\alpha} G\right)$ and $p^{\beta} G=\bigcap_{\alpha<\beta} p^{\alpha} G$ if $\beta$ is a limit ordinal. The height of an element $x$ in $G$ is denoted by $h_{G}(x)$ and is defined to be the largest ordinal $\alpha$ such that $x \in p^{\alpha} G$ if such exists; if not, then $h_{G}(x)=\infty$. We, of course, adopt the convention that $\infty>\alpha$ for all ordinals $\alpha$. It turns out, as usual, in what we do that there is no loss of generality in assuming that $G$ is reduced, that is, that the intersection of all the $p^{\alpha} G$ 's is zero. In this case, $h_{G}(x)=\infty$ if and only if $x=0$. By the height sequence, $S_{G}(x)$, of an element $x$ in $G$ we mean the sequence $\left(h_{G}(x), h_{G}(p x), h_{G}\left(p^{2} G\right), \cdots\right)$, and we specify that $S_{G}(x) \leqq S_{G}(y)$ if and only if $h_{G}\left(p^{n} x\right) \leqq h_{G}\left(p^{n} y\right)$ for each $n \geqq 0$. The group $G$ is said to be transitive if $S_{G}(x)=S_{G}(y)$ implies that there exists an automorphism of $G$ that takes $x$ onto $y$; the condition $S_{G}(x)=S_{G}(y)$ is obviously necessary in order for an automorphism of $G$ to map $x$ onto $y$. If $S_{G}(x) \leqq S_{G}(y)$ implies that there exists an endomorphism of $G$ that maps $x$ onto $y$, then $G$ is called fully transitive.

Kaplansky studied this kind of transitivity in [5]; his statement suggesting that all primary groups might be transitive and fully transitive is well known. Recently, however, Megibben [7] has given an example of a primary group that is neither transitive nor fully transitive. In the positive direction, Griffith [1] has shown that if $p^{\beta} G$ is transitive where $\beta$ is a countable ordinal and if $G / p^{\beta} G$ is a direct sum of countable groups, then $G$ is transitive; a similar result is proved for full transitivity without the countability condition on

Received by the editors June 20, 1968.

1 This research was supported by NSF Grant GP-7442. 
$\beta$. In the present paper, we show that the countability condition on $\beta$ for the transitive case can also be dropped, but more importantly we generalize Griffith's theorems to the case where $G / p^{\beta} G$ is totally projective for any ordinal $\beta$.

A reduced $p$-primary group $G$ is said to be totally projective if $p^{\alpha} \operatorname{Ext}\left(G / p^{\alpha} G, A\right)=0$ for all $\alpha$ and all $A$. Such groups are studied in detail in [8]. It is not difficult to show that any countable reduced primary group $G$ is totally projective by induction on the length of $G$. Thus the direct sum of any number of countable reduced $p$-primary groups is totally projective.

Our main results are based on the next two theorems, which are of independent interest. These theorems are not explicitly stated in [3], but they are essentially contained in the results therein.

Theorem 1 (Hill [3]). Let $G$ be a primary group and let $\beta$ be an ordinal such that $G / p^{\beta} G$ is totally projective. If $K$ and $L$ are finite extensions of $p^{\beta} G$ in $G$, then any height-preserving isomorphism between $K$ and $L$ can be extended to an automorphism of $G$.

Theorem 2 (Hill [3]). Let $G$ be a primary group and let $\beta$ be an ordinal such that $G / p^{\beta} G$ is totally projective. If $K$ is a finite extension of $p^{\beta} G$ in $G$, then any homomorphism from $K$ into $G$ that does not decrease heights, computed in $G$, can be extended to an endomorphism of $G$.

We are now prepared to prove

THEOREM 3. Let $\beta$ be any ordinal. If $p^{\beta} G$ is transitive and if $G / p^{\beta} G$ is totally projective, then $G$ is transitive.

Proof. Let $S_{G}(x)=S_{G}(y)$ and let $n$ be the smallest nonnegative integer such that $p^{n} x \in p^{\beta} G$. Since $S_{G}(x)=S_{G}(y), n$ is also the smallest nonegative integer such that $p^{n} y \in p^{\beta} G$ and $S_{p^{\beta} G}\left(p^{n} x\right)=S_{p^{\beta} G}\left(p^{n} y\right)$. Thus there exists an automorphism $\pi$ of $p^{\beta} G$ that maps $p^{n} x$ onto $p^{n} y$ since $p^{\beta} G$ is transitive. We can extend the map $\pi$ to an isomorphism $\phi$ from $A=\left\{p^{\beta} G, x\right\}$ onto $B=\left\{p^{\beta} G, y\right\}$ by mapping $x$ onto $y$. Moreover, $\phi$ preserves heights computed in $G$, that is, $h_{G}(\phi(a))=h_{G}(a)$ for all $a \in A$. The theorem now follows from Theorem 1 .

In a similar manner, using Theorem 2 , we obtain

THEOREM 4. Let $\beta$ be any ordinal. If $p^{\beta} G$ is fully transitive and if $G / p^{\beta} G$ is totally projective, then $G$ is fully transitive.

By taking $\beta$ sufficiently large in Theorems 3 and 4 , we have the following corollary:

Corollary. If the primary group $G$ is totally projective, then $G$ is both transitive and fully transitive. 
2. A primary group $G$ is potentially transitive if $G /\{x\} \cong G /\{y\}$ provided that $x$ and $y$ are elements of $G$ that have the same height sequence. Obviously, only a potentially transitive group can be transitive. It is the purpose of this section to show that there exist primary groups that are not even potentially transitive.

We begin with the following general result:

THEOREM 5. Let $G$ be a primary group such that $p^{\lambda} G$ is cyclic of order $p$ for some ordinal $\lambda$. Let $T$ be any totally projective group of length $\lambda+1$. In order for $G+T$ to be potentially transitive it must be the case that $G+T^{\prime} \cong G / p^{\lambda} G+T$ for some totally projective group $T^{\prime}$.

Proof. Let $T$ be any totally projective group of length $\lambda+1$ and let $y$ be a nonzero element in $p^{\lambda} T$. Let $p^{\lambda} G=\{x\}$. If $G+T$ is potentially transitive, then

$$
G / p^{\lambda} G+T \cong(G+T) /\{x\} \cong(G+T) /\{y\} \cong G+T /\{y\} .
$$

Denote by $\beta$ the height of $y$ in $T$; actually $\beta=\lambda$. Then $y$ is a direct summand of $p^{\beta} T$; let $p^{\beta} T=\{\boldsymbol{y}\}+K$. We have that $p^{\beta}(T /\{y\})$ $=p^{\beta} T /\{y\}=K$ and $(T /\{y\}) / p^{\beta}(T /\{y\}) \cong T / p^{\beta} T$. Therefore it follows from Proposition 2.6 in [8] that $T /\{y\}$ is totally projective. The proof of the theorem is finished by taking $T^{\prime}=T /\{y\}$.

Using the same method and specializing, we obtain

TheоRем 6. Let $G$ be a reduced primary group such that $p^{\omega} G=Z(p)$, such that $G / p^{\omega} G$ is a closed group, and such that $G$ has a high subgroup that is quasi-closed. Let $H$ be the Prüfer group, that is, let $H$ be the standard totally projective group of length $\omega+1$. Then $G+H$ is not potentially transitive.

REMARK. The existence of a group $G$ satisfying the hypotheses is guaranteed by a theorem of Megibben [6] and a theorem of Hill and Megibben [4].

Proof of Theorem. Let $B=H / p^{\omega} H=\sum Z\left(p^{n}\right)$ and let $C=G / p^{\omega} G$, a closed group. If $G+H$ is potentially transitive, then $G+B=C+H$. Since $C$ is closed and $B$ is a direct sum of cyclic groups, it follows from [2] that $C[p]$ is essentially contained in $G,\left(p^{n} C\right)[p] \subseteq G$ for some positive integer $n$. Thus any high subgroup $M$ of $G$ must contain a nondiscrete subsocle $S$ which is complete in the $p$-adic topology. By hypothesis, $G$ has a high subgroup $M$ that is quasi-closed. We know that $M$ cannot be closed. Furthermore $M$ is pure-complete (every subsocle supports a pure subgroup) [4]. Let $L$ be a pure subgroup of $M$ such that $L[p]=S$. Then $L$ is a closed group since $S$ is complete, and we have $M=A+L$ for some $A$. This leads to a contradiction 
that a quasi-closed group that is not closed is essentially indecomposable [4]. We conclude that $G+K$ is not potentially transitive.

\section{REFERENCES}

1. P. Griffith, Transitive and fully transitive primary abelian groups, Pacific J· Math 25 (1968), 249-254.

2. P. Hill, The isomorphic refinement theorem for direct sums of closed groups, Proc. Amer. Math. Soc. 18 (1967), 913-919.

3. - On the classification of abelian groups (to appear).

4. P. Hill and C. Megibben, Quasi-closed primary groups, Acta Math. 16 (1965), 271-274.

5. I. Kaplansky, Infinite Abelian groups, Univ. of Michigan Press, Ann Arbor, 1954.

6. C. Megibben, On high subgroups, Pacific J. Math. 14 (1964), 1353-1358.

7. - Large subgroups and small homomorphisms, Michigan Math. J. 13 (1966), 153-160.

8. R. Nunke, Homology and direct sums of countable abelian groups, Math. Z. 101 (1967), 182-212.

UNIVERSITY OF HoustoN 